\title{
BILAN DU ZINC 65 ABSORBE PAR VOIE TROPHIQUE CHEZ ANGUILLA ANGUILLA L.
}

\author{
par J.P. BAUDIN ${ }^{1}$
}

Bien que ne faisant pas partie des radionucléides à vie longue, le zinc 65 représente un polluant radioactif important de par sa présence dans les effluents liquides de certaines installations nucléaires et de son isotopie avec un oligoélément biologique essentiel. Dans ce travail l'auteur aborde le problème du transfert et de la concentration de ce radionucléide dans les chaînes alimentaires aquatiques.

L'absorption par des anguilles de zinc 65 contenu dans de la nourriture naturelle se traduit, après l'ingestion de 11 repas échelonnés sur 69 jours, par une accumulation du radionucléide correspondant à un taux de rétention moyen de $22 \%$. Environ $45 \%$ du zinc 65 sont éliminés avec les fèces ce qui représente près de $60 \%$ de 'l'excrétion totale. Entre deux repas successifs on observe une élimination rapide du zinc 65 qui correspond à 2 périodes biologiques courtes, $\mathrm{Tb}_{1} \simeq 1$ jour et $\mathrm{Tb} \simeq 4,5$ jours. Après l'arrêt de l'ingestion de la nourriture contaminée le radionucléide accumulé n'est que très lentement éliminé $\left(\mathrm{Tb}_{3} \simeq 450\right.$ jours) et l'excrétion fécale devient très nettement prépondérante $(\simeq 80 \%$ ). L'organotropisme du zinc 65 se caractérise par une concentration élevée dans les viscères et, en ce qui concerne la distribution, par une forte prédominance du tissu musculaire.

\section{Budget for zinc 65 absorbed through the trophic process in Anguilla anguilla $L$.}

\begin{abstract}
Although it does not belong to the group of radionucleides with a long life, zinc 65 is an important radioactive pollutant because of its presence in liquid effluents from some nuclear installations and because it is isotopic with an essential biological oligoelement. In this work, the author deals with the problems of tranfer and concentration of this radionucleide in the aquatic food chain.

The absorption by the eels of zinc 65 in the natural food appeared, after the ingestion of 11 meals distributed over 69 days, as an accumulation of radionucleide equivalent to a mean retention rate of $22 \%$. About $45 \%$ of the zinc 65 was eliminated with the faeces which represented close to $60 \%$ of the total excretion. Between two successive meals, there was a rapid elimination of zinc 65 and this corresponded to two short biological periods, $\mathrm{Tb}_{1} \simeq 1$ day and $\mathrm{Tb}_{2} \simeq 4,5$ days. After cessation of feeding on contaminated food, the accumulation of radionucleide was eliminated very slowly $\left(\mathrm{Tb}_{3} \simeq 450\right.$ days) and faecal excretion became clearly predominant $(\simeq 80 \%)$. The organotropism of zinc 65 is characterised by a high concentration in the viscera and, from the facts on distribution, by a strong predominance in muscular tissue.
\end{abstract}

1. Chargé de Recherche au C.N.R.S. Laboratoire d'Etudes de Pollution des Eaux, CEA/IPSN/DPr - SERE, CEN Cadarache, 13115 Saint-Paul-lez-Durance. 


\section{INTRODUCTION}

L'ensemble des milieux aquatiques est concerné par la pollution radioactive comme en témoignent les mesures effectuées in situ (Micholet-Cote et al. 1973, Robertson et al. 1973, Foulquier et al. 1979, Jeandel et al. 1980). En conséquence, comme pour les pesticides et les métaux lourds, se trouve posé le problème de la bioamplification des radioisotopes dans les chaînes trophiques et à cet égard, la faune ichtyologique occupe dans les écosystèmes une place prépondérante. C'est dans ce cadre que nous avons réalisé une étude expérimentale consacrée à l'accumulation et à l'élimination du zinc 65 chez Anguilla anguilla, à la suite de l'ingestion d'une nourriture naturelle contaminée.

Les travaux concernant le zinc 65 présentent un intérêt écologique qui revêt plusieurs aspects. Sur le plan strictement radioécologique il a été démontré que ce radionucléide, dont la période est de 245 jours, était présent dans les aires d'épandage des effluents liquides rejetés par certaines installations nucléaires (Foster et Honstead 1967, Cushing et Rose 1970, Scheidauer et al. 1974, Mattson et al. 1980). Cette constatation prend une importance particulière en raison du fait que, par sa présence dans de nombreux enzymes, le zinc est indispensable au déroulement des processus biologiạues (Vallée 1959, Underwood 1971). Mais, oligoélément nécessaire à la vie, le zinc est aussi un agent de pollution chimique dont la toxicité a été mise en évidence par de nombreux travaux (Burton et al. 1972, Matthiessen et Bradfield 1973, Solbe 1974, Chapman 1978, Farmer et al. 1979).

\section{I. - PROTOCOLE EXPERIMENTAL}

Après plusieurs semaines d'acclimatation, 8 anguilles, pesant de $\mathbf{1 5}$ à 30 grammes sont placées dans des bacs individuels contenant 15 litres d'eau maintenue à $20 \pm 1{ }^{\circ} \mathrm{C}$. Cette eau qui provient de l'étang de Citis (Baudin 1981) a une salinité de 12,8\% et une teneur en zinc de $4 \mu \mathrm{g} \cdot 1^{-1}$. Au cours d'une période de 69 jours les anguilles reçoivent un repas hebdomadaire dont la teneur en zinc 65 est préalablement déterminée. Ces repas sont constitués de plusieurs gammares (Gammarus aequicauda Martimov) ayant séjourné durant une semaine dans de l'eau fortement contaminée puis 48 heures dans de l'eau inactive. Lors de chaque repas les poissons absorbent avec la nourriture environ $5 \mu \mathrm{g}$ de zinc stable et de 0,11 à $0,81 \mu \mathrm{Ci}$ de zinc 65 . Entre 2 repas, 3 mesures de l'activité des anguilles sont effectuées, la première inter- 
venant 16 heures après l'ingestion des gammares. Les fèces sont régulièrement recueillies et l'eau des bacs est très fréquemment renouvelée. Après l'ingestion du dernier repas contaminé les mesures ont été poursuivies afin de suivre l'évolution de la teneur en zinc 65 des anguilles et d'évaluer le taux de leur décontamination. La dissection de quelques spécimens à la fin de chacune des deux phases de l'expérience a permis d'étudier l'organotropisme du radiozinc.

Les mesures d'activité nucléaire sont réalisées avec un sélecteur d'amplitude monocanal, dont le dispositif de détection est une sonde puits d'iodure de sodium activé au thallium du type SPT 76. Afin d'assurer une reproductibilité géométrique qui soit satisfaisante les anguilles sont anesthésiées avant d'être introduites dans l'enceinte renfermant la sonde.

\section{2. - RESULTATS}

\subsection{Accumulation du zinc 65}

Pour cette étude nous n'avons pris en compte que les résultats relatifs à 7 anguilles, la huitième n'ayant pas ingéré la totalité de la nourriture fournie.

Les mesures successives font apparaître une évolution discontinue de la radioactivité des poissons liée à l'absorption de zinc 65 suivie de périodes de jeûne au cours desquelles a lieu l'excrétion d'une partie du radionucléide (fig. 1). Un examen détaillé des résultats permet de faire plusieurs constatations.

Tout d'abord on note qui si l'ingestion de chacun des repas se traduit par une augmentation de la radioactivité des anguilles, "le gain " n'est pas proportionnel à la quantié de radiozinc ingéré. Ce phénomène s'explique par l'absence de corrélation entre la teneur en zinc 65 de la nourriture et le taux de rétention du radionucléide (fig. 2). On peut observer que ce paramètre est très différent selon les repas et qu'il varie aussi, notablement, d'un individu à l'autre. En considérant l'ensemble des spécimens et la durée totale de l'étude on constate une diminution continue de ce taux de rétention (Tableau I) dont la valeur moyenne est de $22,4 \%, 7$ jours après l'ingestion du dernier repas. A titre de comparaison signalons que CHIPMAN et al. (1958) indi quent un taux de rétention de $27 \%$ chez Micropogon undulatus 12 heures après l'absorption d'une capsule de gélatine contenant du zinc 65 . Au cours d'une expérience semblable Nakatani et Liu (1964) trouvent une valeur de $13 \%$, après 8 jours, chez Salmo gairdnerii. Enfin Pentreath (1975) cite, pour Pleuronectes platessa, 4 jours après l'inges- 


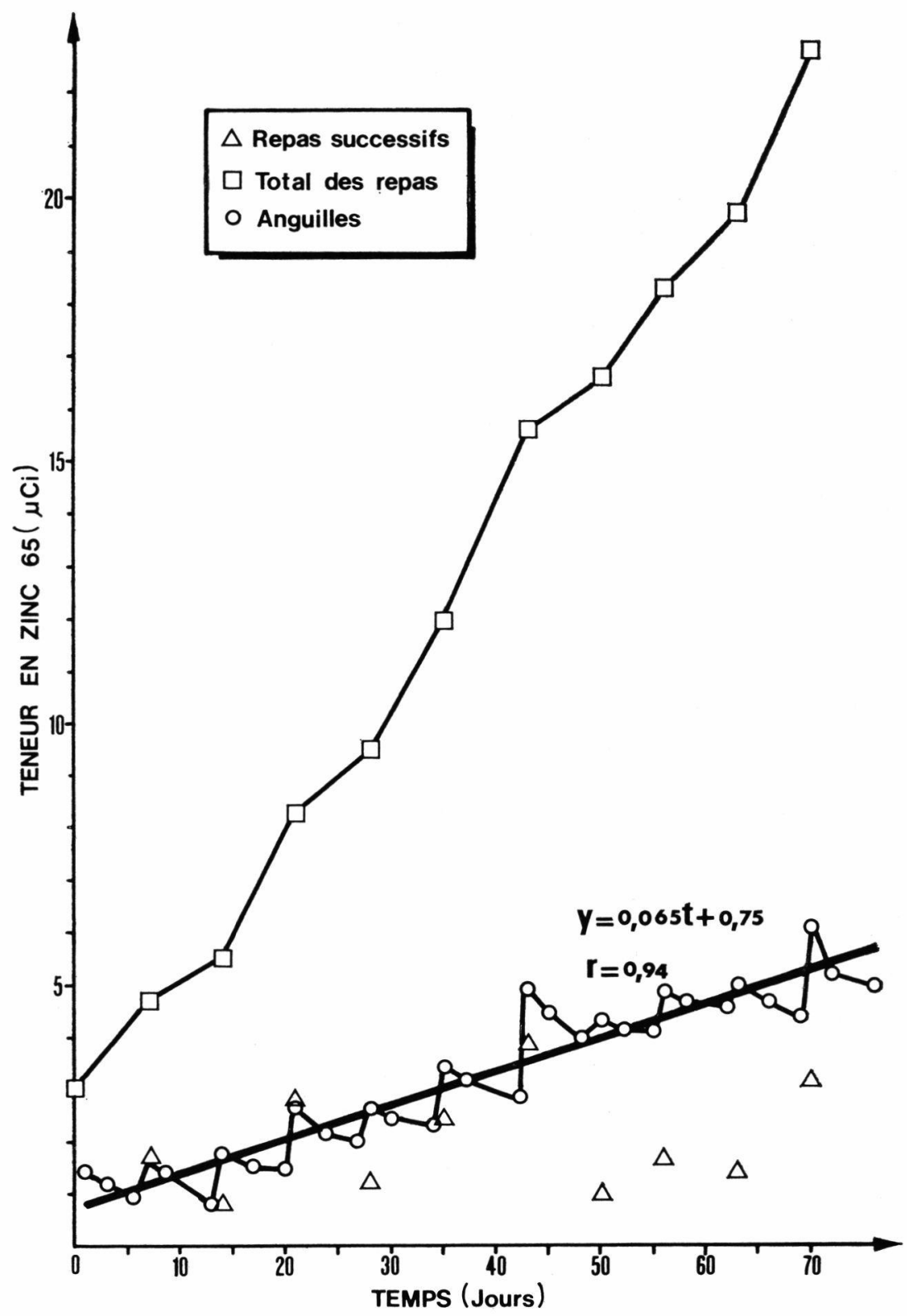

Fig. 1. - Evolution de la radioactivité des anguilles en fonction de la teneur en zinc 65 des repas successifs (sommes des valeurs relatives aux 7 spécimens).

tion, des taux de $72 \%$ dans le cas de capsule de gélatine et de $37,5 \%$ dans le cas de Nereis. A propos de ce dernier exemple on peut remarquer que l'utilisation de supports artificiels ne semble pas souhaitable, 
du moins dans le cas d'un essai d'évaluation des processus se déroulant dans le milieu naturel.

Bien que très irrégulière, l'évolution de la teneur des anguilles en zinc 65 (fig. 1) fait apparaître une accumulation du radionucléide en
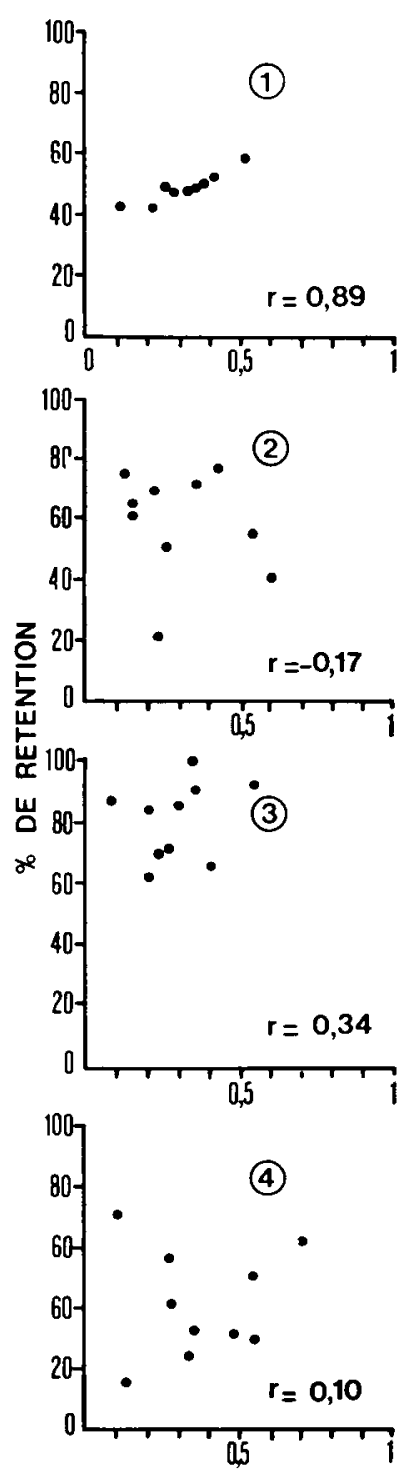

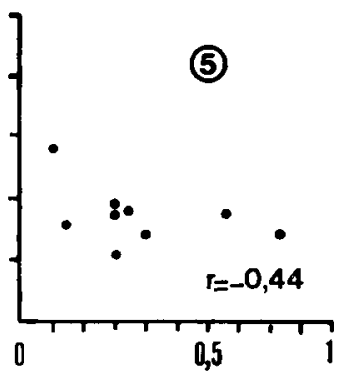

(6)
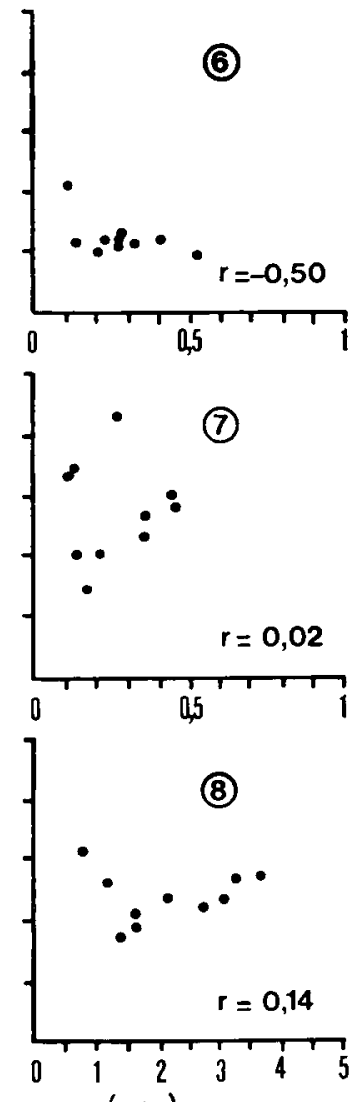

TENEUR EN ZINC 65( $(\mu \mathrm{Ci})$

Fig. 2. - Corrélation entre la teneur en zinc 65 et le taux de rétention du radionucléide: valeurs individuelles (1 à 7$)$ et somme relative aux 7 spécimens ( 8 ). 
TableaU I. - Evolution des taux de rétention et d'excrétion fécale du zinc 65 chez les anguilles durant la phase de "contamination" (valeurs moyennes données au seuil de confiance de $95 \%$ ).

\begin{tabular}{cccc}
\hline $\begin{array}{c}\text { TEMPS } \\
\text { en jours }\end{array}$ & $\begin{array}{c}\text { RÉTENTION } \\
\text { en } \% \text { du zinc 65 ingéré }\end{array}$ & $\begin{array}{c}\text { ExCRÉTION FÉCALE } \\
\text { en } \% \text { du zinc } 65 \text { ingéré }\end{array}$ & en \% du zinc 65 excrété \\
\hline 6 & $34,2 \pm 17,5$ & $29,9 \pm 11,5$ & $44,3 \pm 10,1$ \\
13 & $30,5 \pm 15,5$ & $39,8 \pm 17,9$ & $55,6 \pm 17,5$ \\
20 & $29,5 \pm 13,4$ & $41 \pm 15,7$ & $57,2 \pm 15,9$ \\
27 & $24,8 \pm 11$ & $42,1 \pm 12,9$ & $55,4 \pm 12,5$ \\
34 & $25,1 \pm 11,6$ & $42,4 \pm 12,9$ & $55,8 \pm 11,7$ \\
42 & $24,6 \pm 11,5$ & $43,6 \pm 12,3$ & $57,2 \pm 10,8$ \\
48 & $26,7 \pm 12,7$ & $42 \pm 11$ & $56,7 \pm 8,2$ \\
55 & 26 & $42,3 \pm 10,7$ & $56,7 \pm 8,2$ \\
62 & $25,6 \pm 11,8$ & $43,1 \pm 10,6$ & $57,4 \pm 7,9$ \\
69 & $23,1 \pm 11,1$ & $43,5 \pm 10,5$ & $56 \pm 7,5$ \\
76 & $22,4 \pm 10,6$ & $44,8 \pm 10,2$ & $57,2 \pm 7,4$ \\
\hline
\end{tabular}

fonction du temps. Ce phénomène peut se traduire graphiquement par une droite de régression, calculée par la méthode des moindres carrés, dont l'équation est $\mathrm{y}=0,065 \mathrm{t}+0,75$ dans laquelle y est l'acti vité des poissons (en $\mu \mathrm{Ci}$ ) et $\mathrm{t}$ le moment de la mesure (en Jours). L'accumulation linéaire du zinc 65, observée chez Anguilla anguilla, ist incontestable, cependant la mise en évidence de ce processus, et surtout son évaluation, ne doivent pas être isolées des conditions expérimentales de l'étude. A cet égard les paramètres qui paraissent essentiels concernent la fréquence et le nombre total des repas ainsi que la quantité et la teneur en zinc 65 de la nourriture ingérée.

\subsection{Excrétion du zinc 65}

Deux phases bien distinctes sont à considérer dans l'analyse de ce processus. La première est représentée par la période au cours de laquelle a lieu l'ingestion des repas contaminés ( 0 à 76 jours $)$ et la deuxième commence 7 jours après le dernier apport alimentaire.

Pendant l'intervalle séparant deux repas les anguilles perdent une partie du zinc 65 absorbé avec la nourriture. La quantité éliminée est notablement différente selon les spécimens mais la cinétique de la décontamination est similaire dans tous les cas. L'ensemble des mesures effectuées nous a permis d'évaluer le taux de rétention du radionucléide entre deux repas successifs. Les pourcentages moyens sont de $47,9 \pm 5,4$ après 16 heures, $34,6 \pm 5,7$ après 3 jours et 18,6 \pm 4 après 7 jours. Ces valeurs permettent de déterminer deux périodes biologiques du zinc 65, l'une de 24 heures, l'autre de 4,5 jours ( $f$ ig. 3 ). Considérée sur la durée totale de l'expérience, l'excrétion du zinc 65 augmente légèrement en fonction du temps. Pour compléter le bilan (rappelons que le taux de rétention final est de $22,4 \%$ ) signalons 


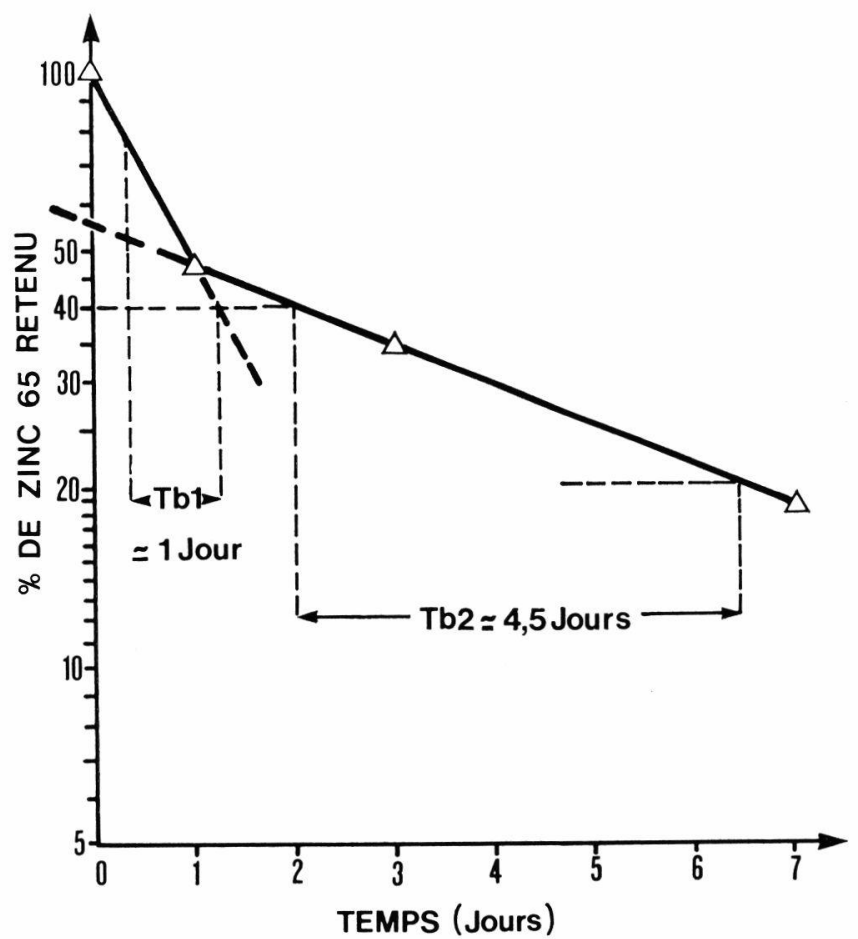

Fig. 3. - Détermination des périodes biologiques du zinc 65 éliminé entre l'ingestion de 2 repas consécutifs.

qu'environ $45 \%$ du zinc 65 absorbé est éliminé avec les fèces ce qui représente près de $60 \%$ de l'excrétion totale (Tableau I).

L'étude de la seconde phase de l'excrétion du zinc 65 a porté sur la fraction du radionucléide retenue par les anguilles 7 jours après l'in-

Tableau II. - Evolution des taux de rétention et d'excrétion fécale du zinc 65 chez les anguilles durant la phase de «décontamination» (valeurs moyennes données au seuil de confiance de $95 \%$ ).

\begin{tabular}{ccc}
\hline $\begin{array}{c}\text { TEMPS } \\
\text { en jours }\end{array}$ & $\begin{array}{c}\text { RÉTENTION } \\
\text { en de la teneur initiale }\end{array}$ & $\begin{array}{c}\text { ExcRÉTION FÉCALE } \\
\text { en du zinc 65 éliminé }\end{array}$ \\
\hline 8 & $95,5 \pm 3,7$ & $81,5 \pm 31,3$ \\
15 & $92,9 \pm 3,6$ & $72,9 \pm 34,6$ \\
22 & $92,9 \pm 3,9$ & $81,9 \pm 31,6$ \\
29 & $93,7 \pm 2,6$ & $88,8 \pm 20,2$ \\
35 & $90,6 \pm 3,1$ & $74,1 \pm 31,8$ \\
42 & $88,9 \pm 3,5$ & $66,2 \pm 29,1$ \\
49 & $90,1 \pm 3,9$ & $76 \pm 32,8$ \\
56 & $85,3 \pm 2,1$ & $57,8 \pm 26,8$ \\
64 & $88,4 \pm 3$ & $73,3 \pm 31,8$ \\
79 & $87,1 \pm 4,3$ & $90 \pm 27,6$ \\
91 & $83,5 \pm 4,7$ & $81,2 \pm 28,3$ \\
98 & $86 \pm 2,9$ & $87,8 \pm 22$ \\
\hline
\end{tabular}


gestion du dernier repas contaminé $(22,4 \%$ du zinc 65 absorbé). L'élimination de ce radiozinc, que l'on peut considéré comme ayant été assimilé est très lente et peu importante. Ainsi après 98 jours la décroissance moyenne de la radioactivité des poissons est de l'ordre de $15 \%$ seulement. Les valeurs du taux de rétention (Tableau II) traduisent une évolution exponentielle simple de ce paramètre. Elles permettent de déterminer, par la méthode des moindres carrés, une courbe dont l'équation est $\mathrm{y}=96,44 \mathrm{e}^{-0,00146 t}$ dans laquelle y représente le taux de rétention du radionucléide et t le temps écoulé depuis le début de l'expérience. D'après l'équation la période biologique du zinc 65 éliminé au cours de la deuxième phase de la décontamination est de l'ordre de 450 jours. A ce propos on peut noter que pour le zinc 65 absorbé directement à partir de l'eau nous avons constaté deux périodes biologiques, la plus longue étant d'environ 120 jours seulement.

En ce qui concerne la voie d'élimination du radionucléide, il appa. raît que l'excrétion fécale qui est importante au cours de la première phase devient ensuite nettement prépondérante (Tableau II).

\subsection{Organotropisme du zinc 65}

Nous avons fondé cette étude de l'organotropisme du zinc $65 \mathrm{chez}$ Anguilla anguilla sur l'évaluation de deux paramètres. Le premier (R) se définit comme le rapport entre la concentration en zinc 65 des organes et celle du poisson in toto (Tableau III). Le second représente la distribution du radionucléide dans l'organisme et s'exprime en pourcentage de la teneur totale (fig. 4).

A la fin de la phase d'accumulation ce sont les viscères qui présentent la teneur en zinc 65 la plus élevée $\left(R_{1}=3,49\right)$. On trouve ensuite les branchies, le squelette, la tête et la peau $\left(R_{1}\right.$ compris entre 1,73 et $1,22)$, puis les muscles et le mucus $\left(R_{1}<1\right)$. Malgré des écarts qui restent parfois importants, les teneurs relatives déterminées après 98 jours d'élimination tendent vers les valeurs calculées pour le zinc stable. D'autre part il apparaît que ces teneurs évoluent différemment selon les organes. Ainsi on constate une nette diminution pour les viscères et le mucus $\left(R_{2}<R_{1}\right)$, une stabilité pour la peau, les branchies et la tête $\left(R_{2} \cong R_{1}\right)$ et une légère augmentation pour les muscles et le squelette $\left(R_{2}>R_{1}\right)$. Il est intéressant de noter que ces deux tissus sont les seuls dont la concentration en zinc stable est proportionnellement plus élevée que celle en zinc 65 (Tableau III).

L'étude de la distribution d'un radioisotope dans un organisme contaminé présente un intérêt particulier dans le domaine de l'évaluation des possibilités de transfert à l'homme des polluants présents dans le milieu par l'intermédiaire des chânes alimentaires. A cet 


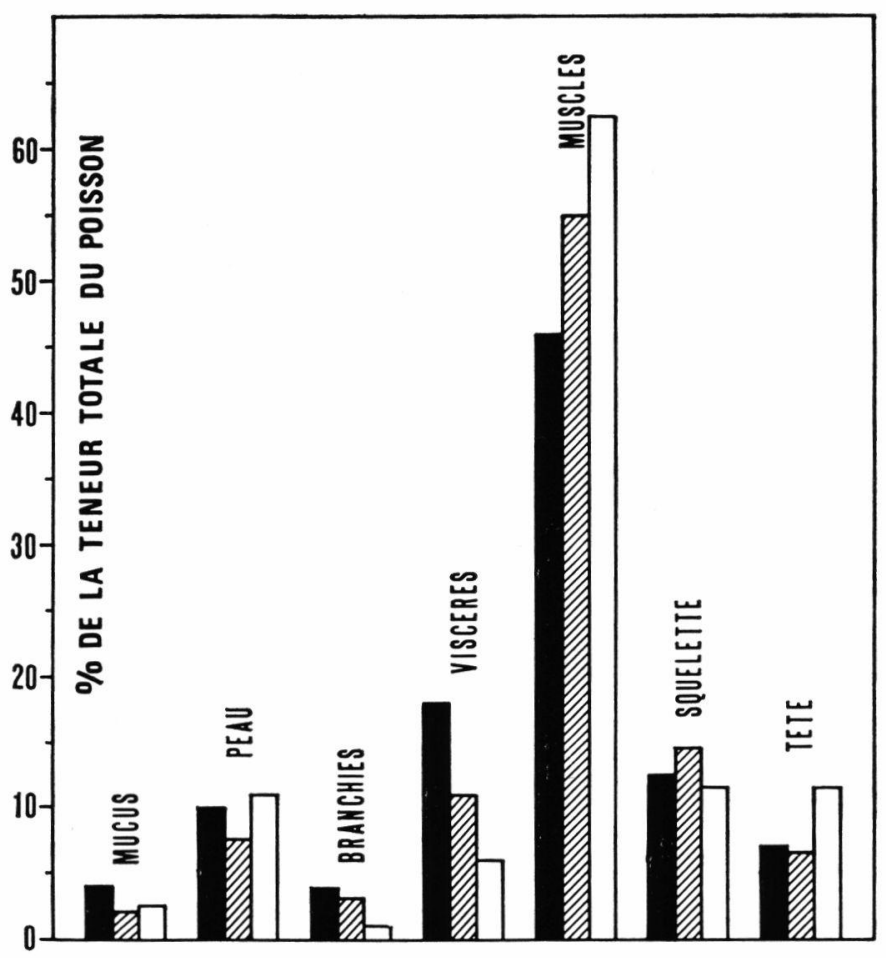

FIg. 4. - Distribution du zinc chez Anguilla anguilla L. (blanc: fin de la phase d'accumulation, gris : fin de la phase d'élimination, noir : zinc stable).

égard, les résultats obtenus (fig. 4) mettent en évidence la place imporpante des muscles qui renferment environ $50 \%$ du zinc 65 accumulé par le poisson. Avec des valeurs un peu différentes, Chipman et al. (1958) chez Micropogon undulatus et Pentreath (1975) chez Pleuronectes platessa ont également observé cette prépondérance du tissu musculaire à la suite de l'absorption du zinc 65 par voie alimentaire.

TABLEAU III. - Concentration en zinc 65 et zinc stable des organes par rapport à celle du poisson in toto.

\begin{tabular}{|c|c|c|c|c|c|c|c|c|}
\hline Organes & & 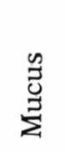 & בٓ & 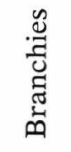 & 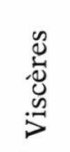 & 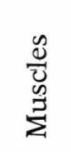 & 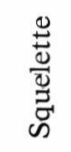 & $\underset{心}{\stackrel{心}{心}}$ \\
\hline $\begin{array}{l}\text { Fin de la phase } \\
\text { d'accumulation }\end{array}$ & $\left(\mathrm{R}_{1}\right)$ & 0,55 & 1,22 & 1,73 & 3,49 & 0,72 & 1,55 & 1,24 \\
\hline $\begin{array}{l}\text { Après } 98 \text { jours } \\
\text { d'élimination }\end{array}$ & $(\mathrm{R})$ & 0,22 & 1,19 & 1,50 & 2,05 & 0,86 & 1,79 & 1,18 \\
\hline Zinc stable & (R) & 0,20 & 0,69 & 1 & 0,98 & 0,95 & 2,31 & 1,08 \\
\hline
\end{tabular}


L'ensemble des données relatives à l'organotropisme du zinc 65 sug. gèrent plusieurs hypothèses, qui ne s'excluent pas nécessairement l'une l'autre, quant aux processus se déroulant au cours de la «phase d'élimination ". On peut penser que la décroissance de la radioactivité totale est due à une décontamination de tous les organes, l'évolution de la teneur relative en radionucléide s'expliquant par des vitesses d'excrétion différentes. Cependant les constatations concernant les muscles et le squelette permettent de penser que l'élimination du zinc 65 peut s'accompagner de la migration et de la redistribution dans l'organisme d'une partie du radionucléide fixé.

\section{3. - CONCLUSION}

Abstraction faite des brusques variations liées au rythme des repas, l'évolution de la radioactivité des anguilles montre que, chez cette espèce, l'ingestion répétée de nourriture contaminée a pour conséquence une accumulation linéaire du radionucléide. Sur le plan de la bioamplification des polluants, le taux de rétention du zinc 65 calculé à l'issue de la "phase d'accumulation " (environ $20 \%$ ) peut être considéré comme élevé. En effet une telle valeur implique, à court terme une concentration de l'élément lors du transfert proie-prédateur. Cependant il faut tenir compte que ce paramètre a été déterminé pour de faibles quantités de nourriture, distribuées au cours de repas espacés, conditions qui peuvent être favorables à une forte rétention du radionucléide.

L'élimination par les poissons du zinc 65 absorbé par voie alimentaire correspond à 3 périodes biologiques très différentes, 24 heures, 4,5 et 450 jours. Ces valeurs permettent d'affirmer que le problème de la vitesse d'excrétion du zinc 65 en fonction du vecteur de la contamination (eau ou nourriture) ne peut être posé sous la forme d'une simple alternative. Pour notre part nous concluons qu'une partie importante du zinc $65(80 \%)$ absorbé par voie trophique est plus rapidement excrétée que le zinc 65 fixé directement à partir de l'eau mais que la fraction restante est au contraire éliminée beaucoup plus lentement.

L'évolution de l'organotropisme du zinc 65 , observée entre les deux phases successives de l'étude, permet de constater que les valeurs calculées pour le radionucléide tendent, en fonction du temps, vers celles déterminées pour l'élément stable. Cette constatation s'applique essentiellement au tissu musculaire dans lequel se trouve accumulé plus de la moitié du zinc 65 total fixé par les anguilles. 


\section{TRAVAUX CITES}

Baudin (J. P.). 1981. - Contribution à l'étude écologique des milieux saumâtres méditerranéens. I : Les principaux caractères physiques et chimiques des eaux de l'étang de Citis. Vie Milieu, 30 (2) : 121-129.

Burton (D. T.), Jones (A. H.) et Cairns (J.). 1972. - Acute zine toxicity to rainbow trout (Salmo gairdnerii) : Confirmation of the hypothesis that dead is related to tissue hypoxia. J. Fish. Res. Bd. Can., $29: 1463-1466$.

Chapman (G. A.). 1978. - Toxicities of cadmium, copper and zinc to four juvenile stages of chinok salmon and steelhead. Trans. Am. Fish. Soc., 107 (6) : 841847 .

Chiman (W. A.), Rice (T. R.) et Price (T. J.). - 1958. - Uptake and accumulation of radioactive zinc by marine plankton, fish and shell-fish. Fish. Bull. U.S. Fish. Widl. Serv., 58 : 279-292.

Cushing (C. E.) et Rose (F. L.). 1970. - Cycling of zinc 65 by Columbia River periphyton in a closed lotic microcosm. Limnol. Oceanogr., 15 (5) : 762-767.

Farmer (G. J.), Ashfield (D.) et Samant (H. S.). 1979. - Effects of zinc on Juvenile atlantic salmon Salmo salar: acute toxicity, food intake, growth and bioaccumulation. Environ. Pollut., 19 (2) : 103-117.

Foster (R. F.) et HonsteAd (J. F.). 1967. - Accumulation of $65 \mathrm{Zn}$ from prolonged consumption of Colombia River fish. Health Phys., 13 (1) : 39-43.

Foulouter (L.), Pally (M.), Descamps (B.), Baudin-Jaulent (Y.) et Lambechts (A.). 1979. - Etude radioécologique du Rhône moyen. II : Essai d'interprétation des mesures de l'activité des poissons. $I I^{*}$ Symposium International de Radioécologie. CEN Cadarache, France : 383-440.

Jeandel (C.), Martin (J. M.) et Thomas (A.). 1980. - Origine et comportement des isotopes du plutonium et du césium 137 dans l'estuaire de la Seine. $C$. $R$. Acad. Sc. Paris, 291, série D : 125-128.

Matthiessen (P.) et Branfield (A. E.). 1973. - The effects of dissolved zinc on the gills of the stickleback, Gasterosteus aculeatus (L.). J. Fish. Biol., 5: 607-613.

Mattson (S.), Fink (R.) et Nilsson (M.). 1980. - Distribution of activation products form barsebäck nuclear power plant (Sweden) in the marine environment. Temporal and spatial variations as established by seaweed. Environ. Pollut., B1 : 105-115.

Micholet-Cote (C. M.), Kirchmann (R.), Cantillon (G.), Colard (J.) et Koch (G.). 1973. - Etude de la radiocontamination des poissons de la Meuse. In " Environmental behaviour of radionuclides released in the nuclear industry. A.I.E.A., Vienne : 413-427.

Nakatani (R. E.) et LIU (D. H. W.). 1964. - Distribution and retention of $65 \mathrm{Zn}$ in trout. Hanford Biology Research Annual Report for 1963, HW. 80500: 109112.

Pentreath (R. J.). 1975. - Radiobiological studies with marine fish. In : "Design of radiotracer experiments in Marine Biological Systems ». A.I.E.A., Vienne: 137-170.

Robertson (D. E.), Silver (W. B.), Langford (J. C.), Pertersen (M. R.) et Perkins (R. W.). 1973. - Transport and depletion of radionuclides in the Colombia River. In "The radioactive contamination of the Marine Environment". A.I.E.A., Vienne : 141-158.

Scheidhauer (J.), Ausset (R.), Planet (J.) et Coulon (R.). 1974. - Programme de surveillance de l'environnement marin du centre de la Hague. In Population dose evaluation and standards for $\operatorname{man}$ and his environment $\%$. A.I.E.A., Vienne : 347-366. 
SolBE (J. F.). 1974. - The toxicity of zinc sulfate to rainbow trout in very hard water. Water Research, 8 : 389-391.

UNDERWOOD (E. J.). 1971. - Zinc. In "Trace elements in human and animal nutrition». New York and London, Academic Press : 543 p.

VAlleE (B. L.). 1959. - Biochemistry, physiology and pathology of zinc. Physiol. Rev., 39 (3) : 443-490. 